\title{
Different Chemotherapy Regimens in the Management of Advanced or Metastatic Urothelial Cancer: a Bayesian Network Meta-Analysis of Randomized Controlled Trials
}

\author{
Yi Wang ${ }^{\mathrm{a}} \quad$ Lingyan Xu $^{\mathrm{b}} \quad$ Xianghu Meng ${ }^{\mathrm{a}} \quad$ Zhiqiang Qin ${ }^{\mathrm{a}} \quad$ Yamin Wanga \\ Chen Chen $^{\mathrm{a}}$ Yichun Wang ${ }^{\mathrm{a}} \quad$ Xiang Zhou $^{\mathrm{a}} \quad$ Qijie Zhang $^{\mathrm{a}} \quad$ Jiadong Xia ${ }^{\mathrm{a}}$ \\ Ninghong Song ${ }^{a}$
}

aDepartment of Urology, The First Affiliated Hospital of Nanjing Medical University, Nanjing, ${ }^{b}$ Department of Oncology, The First Affiliated Hospital of Nanjing Medical University, Nanjing, China

\section{Key Words}

Advanced/metastatic urothelial carcinoma - Chemotherapy - Network meta-analysis • Randomized controlled trials

\begin{abstract}
Background/Aims: Urothelial cancer (UC) as a chemotherapy-sensitive tumor, has achieved remarkable progresses in therapeutic paradigm, particularly in the advanced/metastatic stages. However, both clinicians and patients are confused when it comes to choosing the optimal chemotherapy. Hence, this article was aimed to conduct a comprehensive comparison of different chemotherapy regimens for advanced or metastatic UC in terms of survival benefits or adverse events. Methods: The online databases PubMed, EMBASE and Web of Science were searched systematically and comprehensively for randomized controlled trials (RCTs) up to September 15, 2017. The pooled hazard ratios (HRs) or odds ratios (ORs) with $95 \%$ credible interval (CrI) were calculated by Markov chain Monte Carlo methods. The effectiveness and safety of included regimens were conducted to provide a hierarchy by means of rank probabilities with the help of "R-3.4.0" software and the "gemtc-0.8.2" package. The surface under the cumulative ranking curve (SUCRA) was also incorporated in our analysis for ranking the corresponding chemotherapy regimens. Results: Ten different chemotherapy regimens involved in this article were predominantly of trials in a first-line setting, and eight clinical outcomes were ultimately analyzed in this study. In terms of Overall response rate (ORR), Overall survival (OS) or Progression-free survival (PFS)/Time to progression (TTP), the rank probabilities and SUCRA indicated that Paclitaxel/cisplatin/gemcitabine (PCG) was superior

Y. Wang, L. Xu and X. Meng contributed equally to this work.

\begin{tabular}{ll}
\hline Ninghong Song & Department of Urology, The First Affiliated Hospital of Nanjing Medical University \\
and Jiadong Xia & No. 300 Guangzhou Road, Nanjing, 210029 (China) \\
& Tel. +08613851490672,+08613921433817, E-Mail songninghong_urol@163.com; dongjiaxianjmu@sina.com
\end{tabular}
\end{abstract}




\section{Cellular Physiology Cell Physiol Biochem 2018;50:1-14 \\ \begin{tabular}{l|l} 
DOI: 10.1159/000493951 & $\begin{array}{l}\text { O } 2018 \text { The Author(s). Published by S. Karger AG, Basel } \\
\text { www.karger.com/cpb }\end{array}$
\end{tabular} \\ Wang et al.: Different Chemotherapies in Advanced/Metastatic Urothelial Carcinoma}

to gemcitabine/cisplatin (GC) or methotrexate/vinblastine/doxorubicin/cisplatin (MVAC), the traditional first-line treatment for advanced/metastatic UC. In the case of ORR or PFS/TTP, GC+sorafenib also displayed its superiority in comparison with GC or MVAC. Despite their survival benefits, PCG or GC+sorafenib presented a relatively higher incidence of adverse events. Conclusion: Our results revealed that by adding a paclitaxel or sorafenib into the firstline GC, it could yield a better survival benefit, but also worsen adverse events for advanced/ metastatic UC. Clinically, physicians should weigh the merits of these approaches to maximize the survival benefits of eligible patients.

(C) 2018 The Author(s)

Published by S. Karger AG, Basel

\section{Introduction}

Urothelial cancer (UC) is the most common malignancy of the urinary system, with 58, 950 newly estimated cases and 11, 820 newly estimated deaths in the United States, 2016. According to the Cancer Statistics, it also ranks as the fourth most commonly diagnosed cancer and the eighth leading cause of death in men [1]. Moreover, in terms of advanced/metastatic stages, it accounts for approximately $30 \%$ of patients at initial diagnosis and is considered to be an incurable terminal disease originated from the urinary bladder, renal pelvis, urethra, ureters or prostatic ducts, amongst which, urinary bladder is the most common primary site [2]. Fortunately, it is deemed to be sensitive to chemotherapy. At present, the standard first-line treatment for advanced/metastatic UC is platinum-based chemotherapy-MVAC (methotrexate, vinblastine, doxorubicin, cisplatin) or GC (gemcitabine and cisplatin) regimens. Although MVAC or GC provided a similar median overall survival (OS) in the range of 13 to 15 months and their response rate (RR) was approximately 50\% [3], GC could present a lower pathological response and a lower toxicity in comparison with MVAC [4].

In recent decades, various efforts have been made to improve the efficacy and tolerability profiles of chemotherapy regimens for advanced or metastatic UC. However, their survival benefits remain poor [5]. To gain a higher survival benefit, different combinations of drugs have been reported in accumulating researches, such as adding paclitaxel or monoclonal antibodies or sorafenib to GC, replacing gemcitabine with larotaxel or docetaxel, and so on [6-8]. Currently, chemotherapy regimens for advanced or metastatic urothelial cancer include paclitaxel/cisplatin/gemcitabine (PCG), gemcitabine/cisplatin (GC), gemcitabine/ cisplatin/monoclonal (GC+monoclonal), larotaxel/cisplatin (LC), methotrexate/vinblastine/ doxorubicin/cisplatin (MVAC), docetaxel/cisplatin (DC), carboplatin/paclitaxel (CP), gemcitabine/cisplatin/sorafenib (GC+sorafenib), gemcitabine/carboplatin, cisplatin and so on [6-8]. Along with the introduction and application of these therapeutic options, both clinicians and patients are confused regarding how to choose the optimal therapeutic paradigm. Moreover, randomized controlled trials (RCTs) directly comparing all available treatments were in shortage. Thus, it remains to be completely defined.

Bayesian network meta-analysis, as a powerful and useful approach to combine both direct and indirect evidence, is conducted to provide a hierarchy of the efficacy and safety of different chemotherapy [9-11]. Ten different chemotherapy regimens were ultimately enrolled: PCG, GC, GC+monoclonal, LC, MVAC, DC, CP, GC+sorafenib, gemcitabine/carboplatin, cisplatin. Eight clinical outcomes were finally analyzed: Overall response rate (ORR), OS, Progression-free survival (PFS)/Time to progression (TTP), and adverse events including grade 1-5 Thrombocytopenia, Neutropenia, Anemia, Leukopenia and Diarrhea. The results of our analysis were anticipated to provide some evidence about the cumulative ranking of various chemotherapy regimens in terms of survival outcomes and safety, to guide clinical work. 


\section{Cellular Physiology Cell Physiol Biochem 2018;50:1-14 and Biochemistry Published online: 3 October $2018 \quad \begin{aligned} & \text { DOI: 10.1159/000493951 } 2018 \text { The Author(s). Published by S. Karger AG, Basel } \\ & \text { www.karger.com/cpb }\end{aligned}$ \\ Wang et al.: Different Chemotherapies in Advanced/Metastatic Urothelial Carcinoma}

\section{Materials and Methods}

\section{Search strategy}

The online databases PubMed, EMBASE and Web of Science were searched systematically and comprehensively for RCTs of chemotherapy for advanced or metastatic UC, up to September 15, 2017. The search strategy mainly consisted of three parts (advanced or metastatic urothelial cancer, chemotherapy, and a specific filter for RCTs), using the following keywords in combination with Medical Subject Headings (MeSH) terms and text words: paclitaxel/cisplatin/gemcitabine (PCG), gemcitabine/cisplatin (GC), gemcitabine/cisplatin/monoclonal (GC+monoclonal), larotaxel/cisplatin (LC), methotrexate/vinblastine/ doxorubicin/cisplatin (MVAC), docetaxel/cisplatin (DC), carboplatin/paclitaxel (CP), gemcitabine/ cisplatin/sorafenib (GC+sorafenib), gemcitabine/carboplatin, cisplatin, advanced or metastatic urothelial cancer, and randomized controlled trials (RCTs). Two authors (Y.W and LY.X) independently screened the titles and abstracts, then picked out relevant studies. Later, relevant studies were evaluated according to the selection criteria and all eligible studies were enrolled. If there were duplicated articles, the latest one was enrolled. Any discrepancies were settled by a third reviewer (ZQ.Q).

\section{Selection criteria}

The eligible studies needed to meet the following four inclusion criteria: (1) Randomized controlled trials; (2) Related to advanced or metastatic urothelial cancer; (3) Ten chemotherapy regimens were ultimately enrolled: paclitaxel/cisplatin/gemcitabine (PCG), gemcitabine/cisplatin (GC), gemcitabine/ cisplatin/monoclonal (GC+monoclonal), larotaxel/cisplatin (LC), methotrexate/vinblastine/doxorubicin/ cisplatin (MVAC), docetaxel/cisplatin (DC), carboplatin/paclitaxel (CP), gemcitabine/cisplatin/sorafenib (GC+sorafenib), gemcitabine/carboplatin, cisplatin. At least two chemotherapy regimens were mentioned and compared; (4) Clinical outcomes included overall response rate (responders and sample size), the sample size and hazard ratio (HR) with 95\% confidence interval (CI) of Overall survival (OS) and Progression-free survival (PFS)/Time to progression (TTP) and responders and sample size of different adverse events should be extracted from the enrolled studies. Additionally, the exclusion criteria included the following points: (1) Non-randomized controlled trials; (2) No sufficient and qualified data could be extracted from these studies.

\section{Data extraction and quality assessment}

All data were collected from the original publications including the authors' names, year of publication, treatment regimens, the overall response rate, overall survival, progression-free survival/time to progression and different adverse events. On the basis of the Cochrane Handbook [12], the potential source of bias in the eligible studies was evaluated from the following seven aspects: (1) Random sequence generation; (2) Allocation concealment; (3) Blinding of participants and personnel; (4) Blinding of outcome assessment; (5) Incomplete outcome data; (6) Selective reporting; (7) Other bias. The judgments were graded as a low, high, or unclear risk of bias (http://www.cochrane-handbook.org).

\section{Statistical analysis}

Direct comparison between two different chemotherapy regimens was conducted by traditional pairwise meta-analysis. The pooled hazard ratio (HR) or odds ratio (OR) with $95 \%$ confidence interval (CI) was used to evaluate the direct comparison results. Heterogeneity was assessed by the Chi-square test and I-square test [13]. If Chi-square test $P<0.10$ or $I^{2}>50 \%$, significant heterogeneity existed. In the presence of heterogeneity, the random-effects model was used and HRs or ORs were calculated by the DerSimonian-Laird method. Otherwise, the fixed-effects model and the Mantel-Haenszel method were applied [14]. Begg's and Egger's test were utilized to examine publication bias [15]. $P$ values were adopted by a two-sided test and $P<0.05$ was regarded as statistically significant. In addition, all of the above-mentioned tests of traditional meta-analysis were conducted by Stata software (version 12.0; StataCorp LP, College Station, TX).

Different from traditional pair-wise meta-analysis, a network meta-analysis about multiple treatments was carried out by a random-effect model within a Bayesian framework, by means of the "gemtc-0.8.2" package and R software (version 3.4.0; R Foundation, Vienna, Austria) [16, 17]. Markov chain Monte Carlo methods were applied to calculate hazard ratios (HRs) or odds ratios (ORs) with $95 \%$ credible interval (CrI). The function mtc.run was utilized to generate samples through Markov chain Monte Carlo sampler. 


\section{Cellular Physiology Cell Physiol Biochem 2018;50:1-14 and Biochemistry Published online: 3 October $2018 \quad \begin{aligned} & \text { DOI: } 2018 \text { The Author(s). Published by S. Karger AG, Basel } \\ & \text { www.karger.com/cpb }\end{aligned}$ \\ Wang et al.: Different Chemotherapies in Advanced/Metastatic Urothelial Carcinoma}

Ten thousand simulations were set up initially for each chain as the 'burn-in' period, yielding 40, 000 iterations to get the HRs or ORs of model parameters, while three Markov chains were run simultaneously. The convergence of the model was assessed by the Brooks Gelman Rubin curve method, trace plot and density plot [18]. As a result, the cumulative rank probabilities would provide a hierarchy of each treatment. The matrix and the plot of rank probabilities would be provided by the "gemtc" package simultaneously. Based on the detailed rankings of different chemotherapy regimens, direct and cumulative plots of rank probabilities were established $[19,20]$. The cumulative ranking curve (SUCRA) was also incorporated in our analysis for estimating the probability of a treatment being ranked at a specific place. As for SUCRA, it was a simple transformation of the mean rank, providing a hierarchy of the treatments and accounts for the location and the variance of all relative treatment effects. Meanwhile, for each endpoint, an intervention with a higher SUCRA value was considered more desirable than others [21,22]. Depending on the results of rank probabilities and SUCRA, we could easily find the ranking of each chemotherapy regimen [23].

The pooled HRs or ORs from network or traditional pair-wise meta-analysis were compared to estimate the consistency. When a loop connecting three arms existed, node-splitting method was utilized to separate the evidence concerning certain comparison into direct and indirect evidence to evaluate the inconsistency by reporting its Bayesian P value [24]. Lastly, the mtc.anohe command of the "gemtc" package was applied to assess the global heterogeneity, by means of heterogeneity variance parameter $I^{2}$.

\section{Results}

\section{Search results and study characteristics}

A total of 336 relevant studies were comprehensively identified through online databases by previous search strategy. After screening the titles and abstracts, 289 records were excluded because of duplicates, or because they were unrelated to chemotherapy regimens or advanced/metastatic UC. The full texts of the remaining 47 articles were evaluated by the reviewers. Thirty-one relevant studies were removed as a result of reviews, not RCTs, metaanalysis or lack of sufficient data. Finally, 16 RCTs were eligible for this meta-analysis and the quality of evidence was assessed by the Cochrane Handbook, which graded each potential source of bias as low, high or unclear. Their results of each risk of bias were presented in Fig. 1 and Fig. 2. Moreover, the flow diagram of the literature selection process was displayed in Fig. 3 [25-40].

The detailed information about the enrolled 16 studies with 3, 196 patients was displayed in Supplementary Table S1 and Supplementary Table S2 (for all supplementary material see www.karger.com/10.1159/000493951/). This study covered 10 different chemotherapy regimens: PCG, GC, GC+monoclonal, LC, MVAC, DC, CP, GC+sorafenib, gemcitabine/carboplatin, cisplatin; and eight clinical outcomes: ORR, OS, PFS/TTP as well as adverse events including grade 1-5 Thrombocytopenia, Neutropenia, Anemia, Leukopenia and Diarrhea. Meanwhile, these 10 different chemotherapy regimens were predominantly of trials in a first-line setting. The network structure diagrams were detailed in Fig. 4, which was

Fig. 1. Risk of bias summary. Review author's judgements for each risk of bias item for each included study.

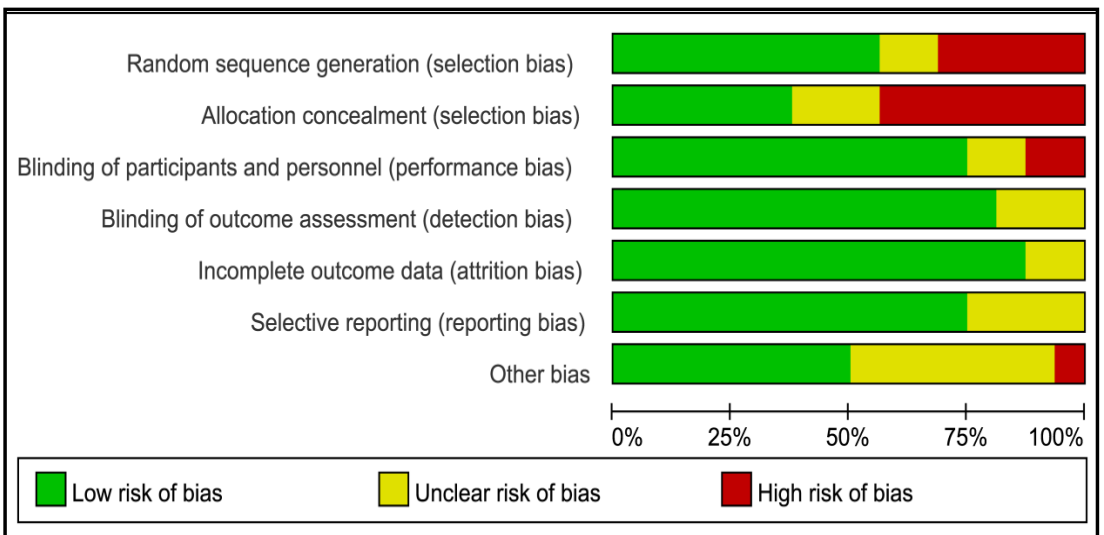


the reflection of the relationships of different chemotherapy regimens. Meanwhile, the number of comparisons was expressed proportionally by the thicknesses of the lines, and the number of treatments was reflected proportionally in the diameters of the circles. Trace plot and density plot were utilized to access the convergence of the model by the Brooks Gelman Rubin curve method.

\section{Overall response rate (ORR)}

A total of 14 studies including 10 chemotherapy regimens (PCG, GC, GC+monoclonal, LC, MVAC, DC, CP, GC+sorafenib, gemcitabine/carboplatin and cisplatin) were involved in the analysis of ORR. Their relationship of different chemotherapy regimens was detailed in Fig. $4 \mathrm{~A}$ and the response rate of different chemotherapy regimens listed by ORs and corresponding 95\% CrIs were presented in Supplementary Fig. S1A. However, they were not statistically significant. Fig. 5A was a direct plot of rank probabilities, from which we could easily find the ranking of each chemotherapy regimens. Fig. $6 \mathrm{~A}$ was a cumulative rank plot with the surface under the cumulative ranking curve (SUCRA) of each intervention and its detailed values were presented in Table 1. Meanwhile, the higher the SUCRA value, the higher possible ranking was that of the treatment. As indicated by the results of rank probabilities and SUCRA, the chemotherapy regimens from best to worst were PCG, GC+sorafenib, GC, GC+monoclonal, MVAC, CP, gemcitabine/ carboplatin, LC, DC and cisplatin respectively. Owing to the absence of a loop connecting three arms, the node-splitting method could not be applied.

\section{Overall survival (OS)}

Fifteen studies were recruited to compare OS between 10 chemotherapy regimens (PCG, GC, GC+monoclonal, LC, MVAC, DC, CP, $\mathrm{GC}+$ sorafenib, gemcitabine/carboplatin and

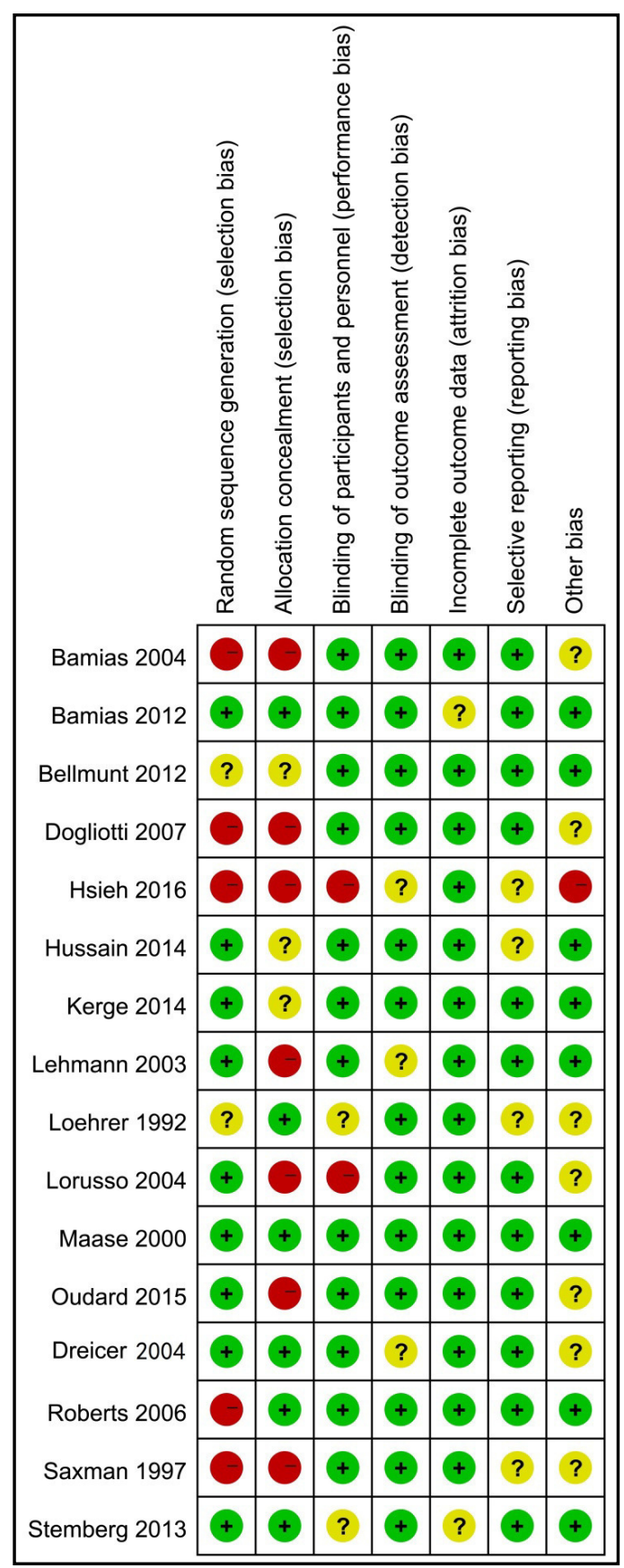

Fig. 2. Risk of bias graph. Review author's judgements for each risk of bias item are presented as percentages across all included studies. cisplatin). Their relationship to different chemotherapy regimens was detailed in Fig. 4B and the pooled HRs and corresponding 95\% CrIs of OS were displayed in Supplementary Fig. S1B. As indicated in the results, PCG, GC and MVAC showed a better OS, compared with gemcitabine/carboplatin (HR=0.44, 95\% $\mathrm{CrI}$ 0.21-0.94; HR=0.51, 95\% CrI 0.26-1.0; HR=0.49, 95\% CrI 0.24-0.99, respectively). PCG, GC and MVAC also displayed a better OS, compared with cisplatin (HR=0.55, 95\% CrI 0.36-0.89; $\mathrm{HR}=0.64,95 \% \mathrm{CrI} 0.47-0.91$; $\mathrm{HR}=0.62,95 \% \mathrm{CrI} 0.48-0.80$, separately). As indicated by the results of rank probabilities and SUCRA, PCG, MVAC, GC+monoclonal, GC, GC+sorafenib, CP, 
LC, DC, cisplatin and gemcitabine/carboplatin indicated a decreasing tendency of the OS (Fig. 5B, Fig. 6B, Table 1). The node-splitting method was omitted, because of the absence of a loop connecting three arms.

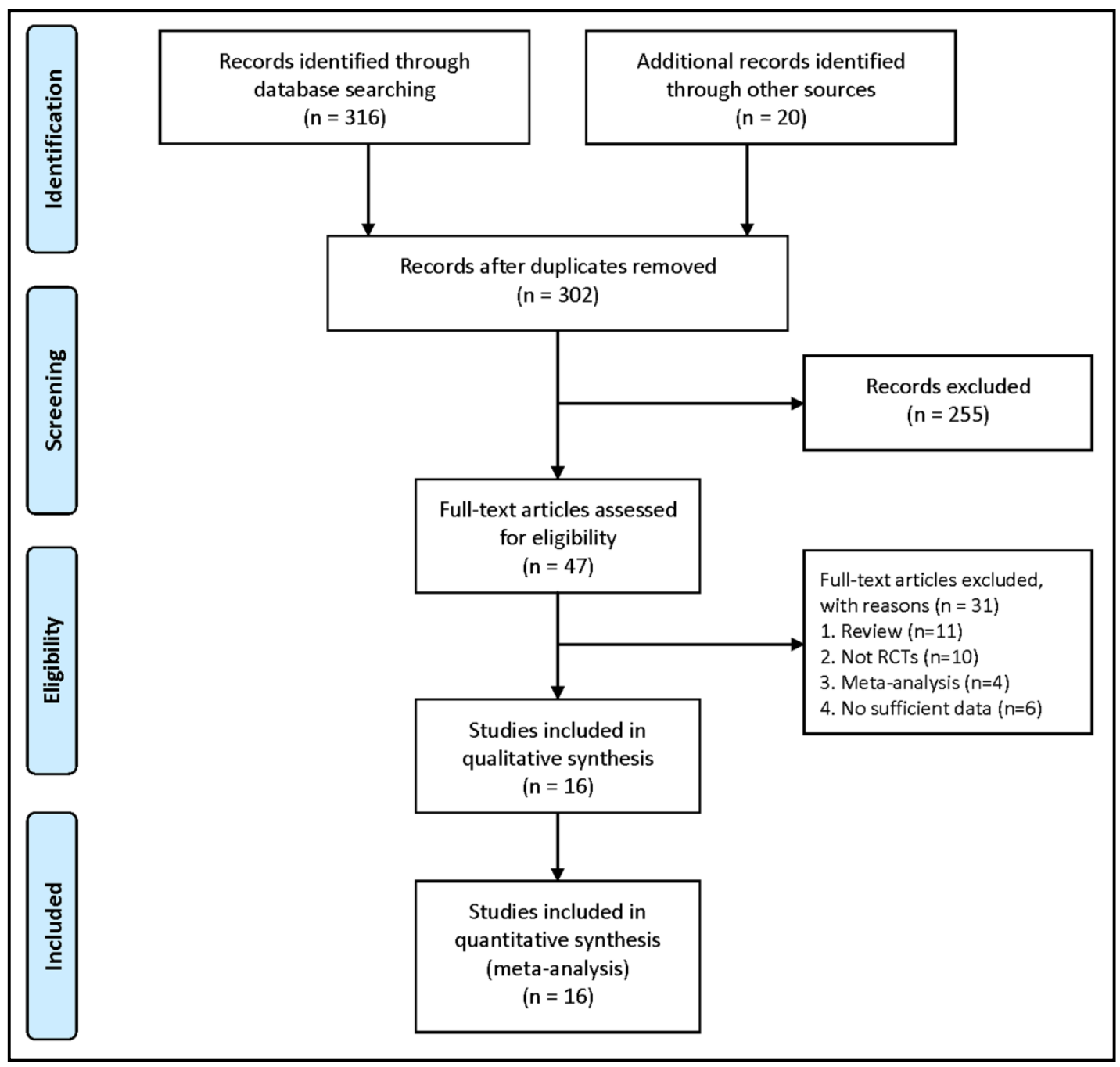

Fig. 3. The flow diagram of the literature selection process.

Table 1. The SUCRA probabilities of different chemotherapy regimens for advanced/metastatic urothelial carcinoma on clinical outcomes. SUCRA: the value of surface under the cumulative ranking curve (The higher the SUCRA value, the higher possible ranking was that of the treatment.); ORR: Overall response rate; OS: Overall survival; PFS/TTP: Progression-free survival/Time to progression; PCG=paclitaxel/cisplatin/ gemcitabine; $\quad \mathrm{GC}=$ gemcitabine/cisplatin; monoclonal=trastuzum/cetuximab; $\mathrm{MVAC}=$ methotrexate/ vinblastine/doxorubicin/cisplatin; LC=larotaxel/cisplatin; $\quad \mathrm{DC}=$ docetaxel/cisplatin; $\quad \mathrm{CP}=$ carboplatin/ paclitaxel; GC+sorafenib=gemcitabine/cisplatin/sorafenib; G+Carboplatin= gemcitabine/carboplatin

\begin{tabular}{|c|c|c|c|c|c|c|c|c|c|c|}
\hline Intervention & PCG & GC & GC+Monoclonal & LC & MVAC & DC & $\mathrm{CP}$ & G+Carboplatin & GC+Sorafenib & Cisplatin \\
\hline ORR & $80.25 \%$ & $66.50 \%$ & $56.53 \%$ & $38.82 \%$ & $55.36 \%$ & $25.50 \%$ & $44.65 \%$ & $44.63 \%$ & $71.74 \%$ & $11.02 \%$ \\
\hline Overall survival & $78.89 \%$ & $61.61 \%$ & $66.21 \%$ & $42.09 \%$ & $69.41 \%$ & $38.70 \%$ & $44.79 \%$ & $14.08 \%$ & $59.79 \%$ & $19.42 \%$ \\
\hline PFS/ TTP & $78.90 \%$ & $65.76 \%$ & $59.71 \%$ & $22.64 \%$ & $64.53 \%$ & $24.83 \%$ & $34.89 \%$ & $61.91 \%$ & $67.94 \%$ & $13.89 \%$ \\
\hline Thrombocytopenia & $71.01 \%$ & $76.30 \%$ & $60.65 \%$ & $23.50 \%$ & $47.46 \%$ & $17.79 \%$ & $32.34 \%$ & $77.31 \%$ & $64.18 \%$ & $24.45 \%$ \\
\hline Neutropenia & $60.06 \%$ & $41.74 \%$ & $36.74 \%$ & $11.99 \%$ & $60.13 \%$ & $32.05 \%$ & $16.24 \%$ & $56.05 \%$ & - & - \\
\hline Anemia & $54.08 \%$ & $64.93 \%$ & $57.54 \%$ & $55.25 \%$ & $42.18 \%$ & $36.88 \%$ & $88.49 \%$ & $69.93 \%$ & $59.06 \%$ & $46.30 \%$ \\
\hline Leukopenia & $52.76 \%$ & $45.34 \%$ & $51.11 \%$ & $24.50 \%$ & $42.50 \%$ & - & - & $54.35 \%$ & $33.31 \%$ & $11.12 \%$ \\
\hline Diarrhea & $28.07 \%$ & $18.70 \%$ & $40.60 \%$ & $54.41 \%$ & $48.62 \%$ & $34.02 \%$ & - & $30.90 \%$ & $59.67 \%$ & - \\
\hline
\end{tabular}


Cellular Physiology Cell Physiol Biochem 2018;50:1-14

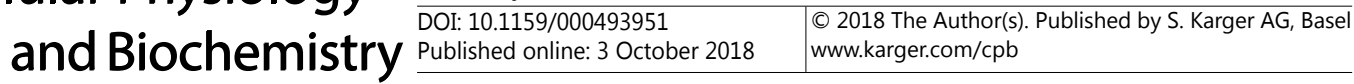

Wang et al.: Different Chemotherapies in Advanced/Metastatic Urothelial Carcinoma

Fig. 4. Network structure diagrams. (A) Overall response rate (ORR); (B) Overall survival (OS); (C) Progression-free survival (PFS)/ Time to progression (TTP);

(D) Thrombocytopenia; (E) Neutropenia; (F) Anemia; (G) Leukopenia; $\quad(\mathrm{H})$ Diarrhea. The thicknesses of the lines were proportional to the number of c o m paris on s; The diameters of the circles were proportional to the number of treatments.

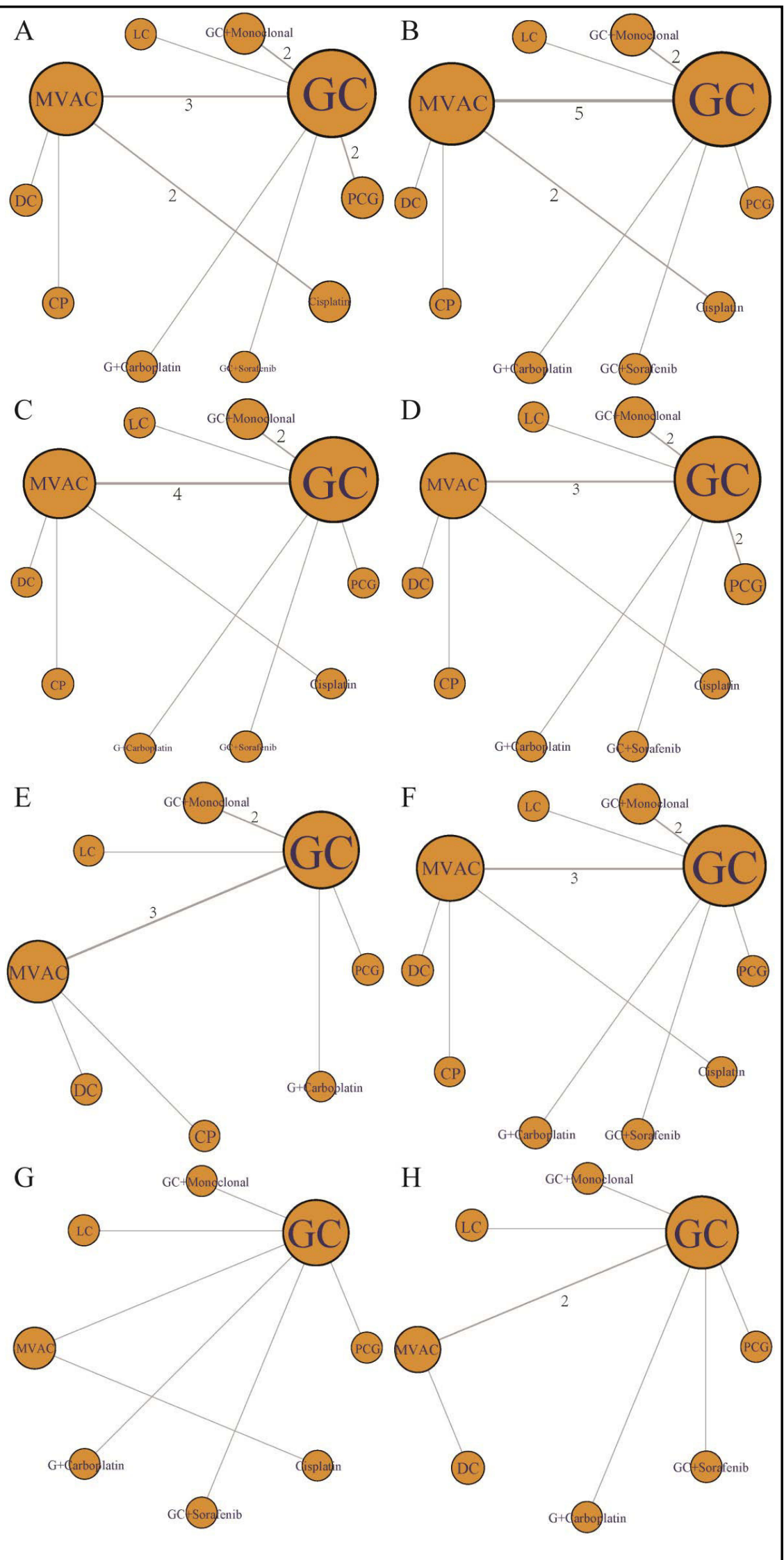




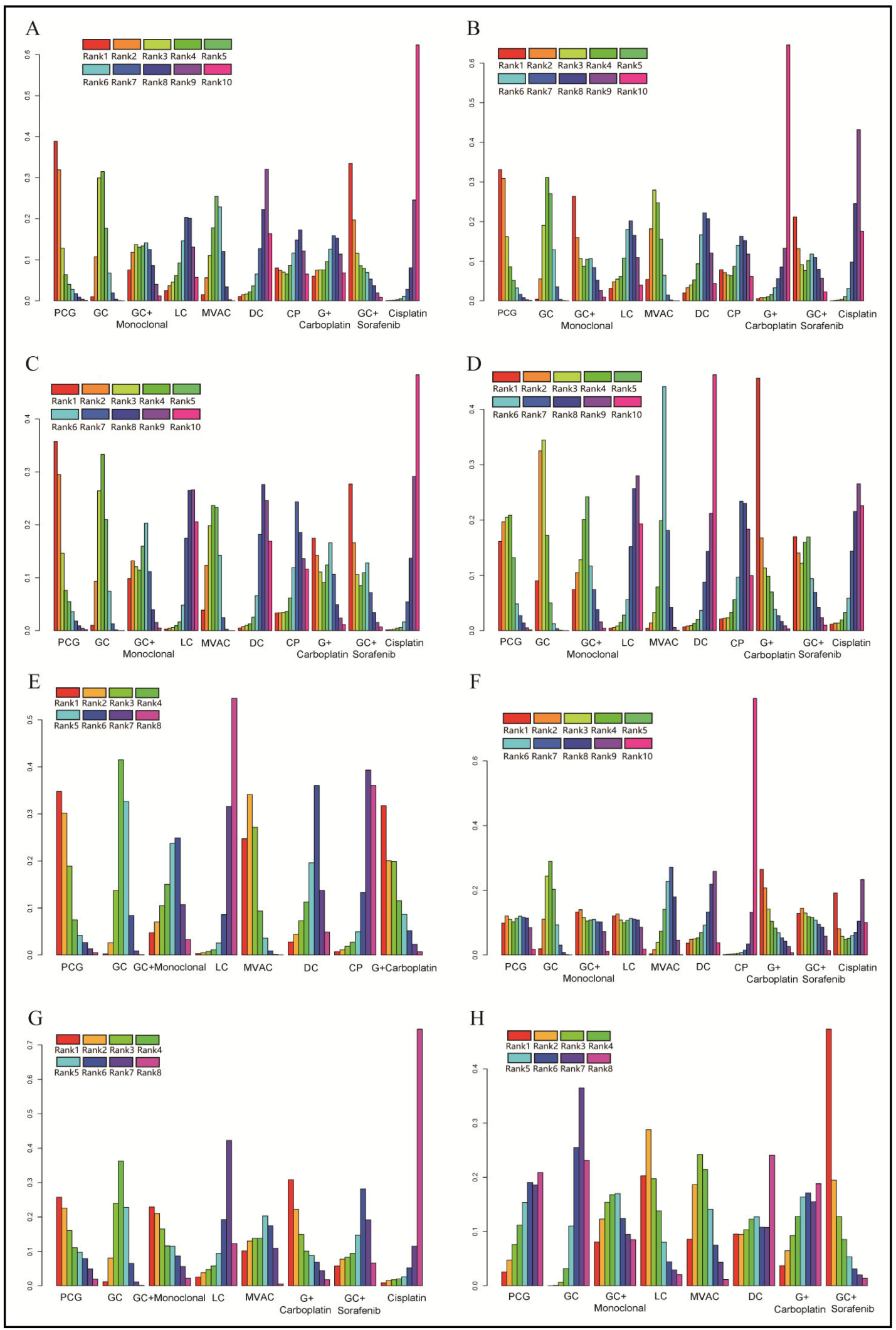

Fig. 5. Rank probabilities of the efficacy and tolerability of different chemotherapy regimens. (A) Overall response rate (ORR); (B) Overall survival (OS); (C) Progression-free survival (PFS)/Time to progression (TTP); (D) Thrombocytopenia; (E) Neutropenia; (F) Anemia; (G) Leukopenia; (H) Diarrhea. 


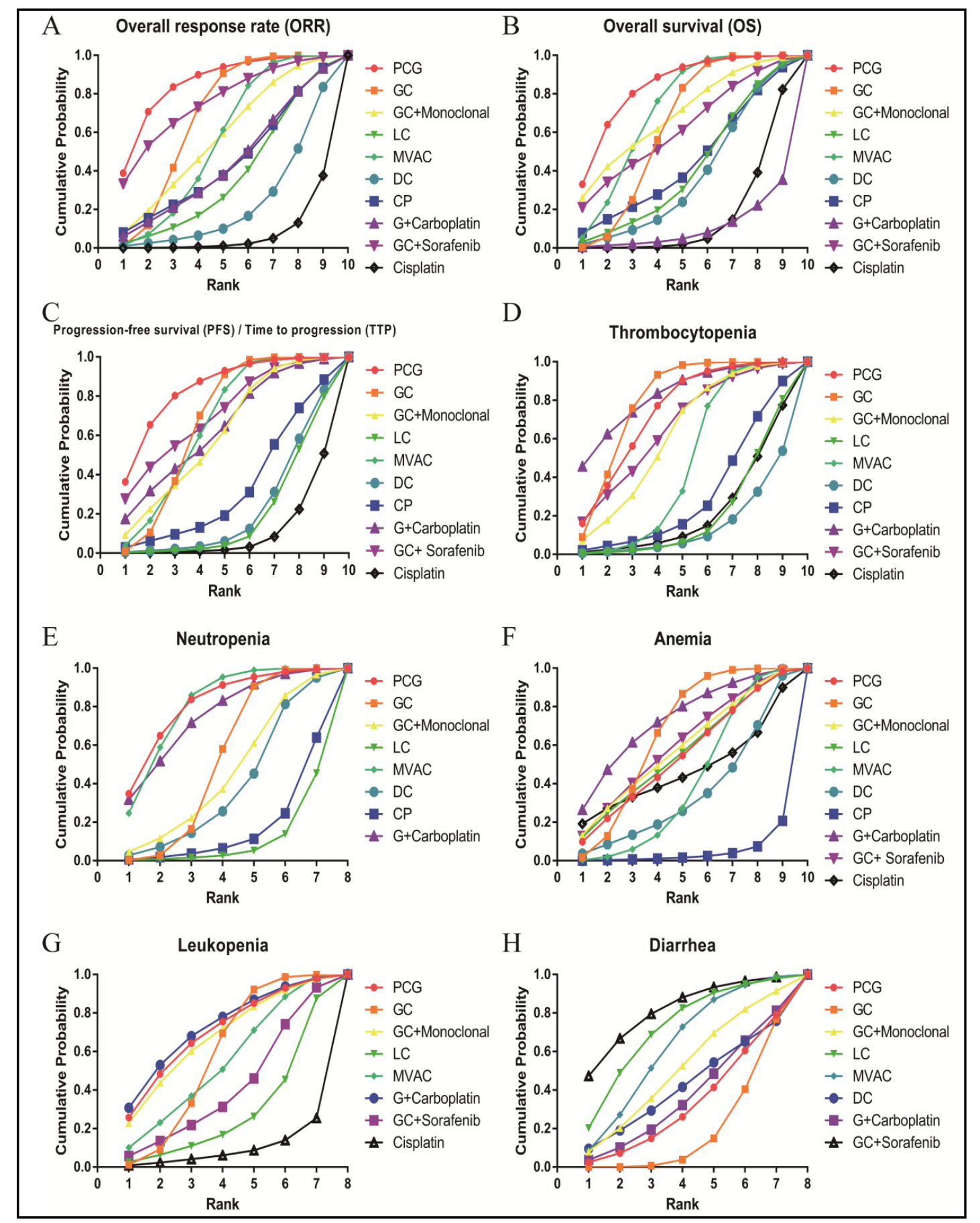

Fig. 6. Cumulative rank plot with the surface under the cumulative ranking curve (SUCRA) of each intervention for effective outcomes. (A) Overall response rate (ORR); (B) Overall survival (OS); (C) Progression-free survival (PFS)/Time to progression (TTP); (D) Thrombocytopenia; (E) Neutropenia; (F) Anemia; (G) Leukopenia; (H) Diarrhea.

\section{Progression-free survival (PFS) / Time to progression (TTP)}

The results of PFS/TTP were calculated by 13 studies including 10 chemotherapy regimens (PCG, GC, GC+monoclonal, LC, MVAC, DC, CP, GC+sorafenib, gemcitabine/carboplatin and cisplatin). Their relationship of different chemotherapy regimens was detailed in Fig. 4C and the efficacy of different therapeutic paradigms for HRs and corresponding 95\% CrIs was 


\section{Cellular Physiology Cell Physiol Biochem 2018;50:1-14 \\ \begin{tabular}{l|l} 
DOI: 10.1159/000493951 & $\begin{array}{l}\text { O } 2018 \text { The Author(s). Published by S. Karger AG, Basel } \\
\text { www.karger.com/cpb }\end{array}$
\end{tabular} \\ Wang et al.: Different Chemotherapies in Advanced/Metastatic Urothelial Carcinoma}

detailed in Supplementary Fig. S1C. As indicated in the result, PCG and GC showed a better survival benefit, compared with LC (HR=0.52, $95 \%$ CrI 0.28-0.98; HR=0.60, $95 \%$ CrI 0.370.96, separately); PCG, GC and MVAC displayed a longer PFS/TTP, compared with cisplatin (HR=0.45, 95 \% CrI 0.23-0.87; HR=0.52, 95 \% CrI 0.32-0.85; HR=0.52, 95 \% CrI 0.34-0.81, respectively). As indicated by the results of rank probabilities and SUCRA, the chemotherapy regimens from best to worst were PCG, GC+sorafenib, GC, MVAC, gemcitabine/carboplatin, $\mathrm{GC}+$ monoclonal, $\mathrm{CP}, \mathrm{DC}, \mathrm{LC}$ and cisplatin indicating a decreasing tendency of the OS (Fig. 5C, Fig. 6C, Table 1). Due to the absence of a loop connecting three arms, the node-splitting method was not calculated.

\section{Adverse events (Thrombocytopenia, Neutropenia, Anemia, Leukopenia, Diarrhea)}

Five adverse events including grade 1-5 Thrombocytopenia, Neutropenia, Anemia, Leukopenia and Diarrhea were ultimately analyzed in this article. Their relationship of different chemotherapy regimens was detailed in Fig. 4D-4H and the ORs and corresponding 95\% CIs of each adverse event were shown in Supplementary Fig. S1D-H. The adverse events of Thrombocytopenia were referred in all 16 eligible studies with 10 chemotherapy regimens and the rank of the possible frequency from highest to lowest was gemcitabine/ carboplatin, GC, PCG, GC+sorafenib, GC+monoclonal, MVAC, CP, cisplatin, LC and DC (Fig. 5D, Fig. 6D, Table 1). The side effect of Neutropenia was analyzed according to 13 relative studies with eight chemotherapy regimens. Meanwhile, MVAC, PCG, gemcitabine/carboplatin, GC, $\mathrm{GC}+$ monoclonal, DC, CP and LC had a decreasing possibility to magnify the problem (Fig. 5E, Fig. 6E, Table 1). The results of Anemia were calculated according to 15 eligible studies with 10 chemotherapy regimens. Meanwhile, CP, gemcitabine/carboplatin, GC, GC+sorafenib, GC+monoclonal, LC, PCG, cisplatin, MVAC and DC had a decreasing frequency to appear anemic (Fig. 5F, Fig. 6F, Table 1). Leukopenia was reported in 10 studies including eight different chemotherapy regimens and gemcitabine/carboplatin, PCG, GC+monoclonal, GC, MVAC, GC+sorafenib, LC and cisplatin indicated the decreasing rank of leukopenia (Fig. 5G, Fig. 6G, Table 1). Diarrhea was analyzed in 11 recruited studies including eight chemotherapy regimens. GC+sorafenib, LC, MVAC, GC+monoclonal, DC, gemcitabine/carboplatin, PCG and GC indicated a decreasing tendency (Fig. 5H, Fig. 6H, Table 1).

\section{Discussion}

Urothelial cancer (UC) is the most common malignancy of the urinary system in the male population. As for advanced or metastatic UC, it is an incurable terminal disease and accounts for approximately $30 \%$ of patients at initial diagnosis. Fortunately, it is a chemotherapysensitive tumor and platinum-based chemotherapy remains the standard treatment [38, 41]. In recent decades, great efforts have been made to improve the efficacy and tolerability profiles of standard first-line treatment of advanced/metastatic UC (MVAC or GC). However, their survival benefits remained poor. In terms of therapeutic options, both clinicians and patients were confused regarding the choice of the most ideal therapeutic paradigm. Moreover, due to the shortage of sufficient information about the comprehensive comparison of different chemotherapy regimens, this network meta-analysis was implemented to clarify the confusion.

As far as we were concerned, this was the first network meta-analysis comparing the efficacy and safety of different chemotherapy regimens in the treatment of advanced/ metastatic UC. A total of 10 different chemotherapy regimens were enrolled in this article: PCG, GC, GC+monoclonal, LC, MVAC, DC, CP, GC+sorafenib, gemcitabine/carboplatin, and cisplatin. In addition, eight clinical outcomes (ORR, OS, PFS/TTP and five adverse events including Thrombocytopenia, Neutropenia, Anemia, Leukopenia and Diarrhea) were ultimately analyzed to provide a hierarchy of the effectiveness and safety of included regimens. Our results of the rank probabilities and SUCRA indicated that in terms of ORR, OS or PFS/TTP, PCG was superior to GC or MVAC, the traditional first-line treatment for advanced/metastatic 


\section{Cellular Physiology Cell Physiol Biochem 2018;50:1-14 and Biochemistry Published online: 3 October $2018 \quad \begin{aligned} & \text { DOI: 10.1159/000493951 } 2018 \text { The Author(s). Published by S. Karger AG, Basel } \\ & \text { www.karger.com/cpb }\end{aligned}$ \\ Wang et al.: Different Chemotherapies in Advanced/Metastatic Urothelial Carcinoma}

UC. Moreover, in the case of ORR or PFS/TTP, GC+sorafenib also displayed its superiority in comparison with GC or MVAC. However, their incidence rates of adverse events were higher.

At the beginning of the chemotherapy of UC, from single-drug cisplatin to a combination of four drugs, MVAC had superior results in phase III studies. However, MVAC was associated with significant toxicity, as well as a toxic related death rate of 3-4\% [39]. When GC was put forward, there were a lot of trials designed to compare therapeutic effects of both schemes. With respect to the results of these trials, although patients had similar survival advantages, GC had a better safety profile and tolerability [33, 37, 38]. Subsequently in a phase III trial by Bellmunt et al. [30], a large number of patients were compared with different clinical antitumor activity by utilizing GC with or without paclitaxel and their results suggested that the three-drug regimen could provide a significantly higher response rate $(55.5 \%$ vs $43.6 \%$, $p=0.0031$ ) and a prolonged 3.1 month survival, but with no statistical significance. When assessing the safety of the two regimens, these treatments were well tolerated with more febrile neutropenia on PCG than GC $(13.2 \%$ vs $4.3 \%, p<0.001)$ and more thrombocytopenia and bleeding on GC than PCG(11.4\% vs 6.8\%, $p=0.05)$ [30]. Moreover, a meta-analysis by Giannatempo et al. disclosed that adding taxanes (paclitaxel or docetaxel only) to gemcitabine/platinum for the first-line therapy of advanced or metastatic UC displayed a trend for improved OS and higher grade $\geq 3$ neurotoxicity [42]. In addition, similar results could be found in another meta-analysis indicating that gemcitabine with taxanes could be an alternative to gemcitabine plus carboplatin for advanced cisplatin-ineligible urothelial cancer [43].

Abovementioned results were in line with ours and our results revealed that by adding a paclitaxel or sorafenib into the first-line GC, it could yield a better survival benefit, but worsen adverse events for advanced/metastatic UC. In a comprehensive consideration of the survival benefits and the adverse events, GC or MVAC remained the mainstay of chemotherapy regimens. Clinically, physicians should weigh the merits of these approaches to maximize the survival benefit of eligible patients. Larger sample sizes of strictly designed RCTs were required to validate our findings.

Despite their survival benefits, the adverse effects of PCG or GC+sorafenib were not rarely demonstrated in this article. As indicated by our results, the predominant adverse events of PCG were thrombocytopenia, neutropenia and leukopenia and major side effects of $\mathrm{GC}+$ sorafenib were thrombocytopenia and diarrhea. Although our results provided a useful train of thought for developing the next generation of chemotherapy drugs, more attention should be paid to the increased incidence of adverse events rather than merely focusing on the survival benefits.

The strength of this study was mainly that it was the first time for us to put forward the hierarchy of different chemotherapy regimens, which could guide the clinical work. In addition, all of the included articles were RCTs, which could have a clear impact on the group baseline features and provide enough statistical power. Nevertheless, there were several potential limitations in the meta-analysis simultaneously. First, the addition of paclitaxel to GC showed better clinical benefits, but there were only two trials comparing the efficacy and safety of PCG and GC. Second, not all reported adverse events were evaluated in the analysis. Third, inconsistencies in the study population, intervention, design and evaluation of the curative effect of the entire enrolled trials, could lead to some potential confounding and bias. Fourth, due to the absence of sufficient data on monoclonal antibodies, we had difficulty in explaining them separately. Moreover, it was more easily associated with leading to bias, when they were analyzed independently. Meta-analysis, as a powerful tool, could provide more reliable results than a single study by combining all eligible studies [44]. As a reminder for subsequent researches, larger samples with more strictly designed RCTs regarding monoclonal antibodies with different targets were required to verify our findings. Lastly, some trails didn't provide direct HR of OS or PFS/TTP. Extracting data from KaplanMeier curves might result in a potential bias of the final conclusion.

Everything considered, the present network meta-analysis as a useful tool in combining both direct and indirect evidence revealed that adding a paclitaxel or sorafenib into the 


\section{Cellular Physiology Cell Physiol Biochem 2018;50:1-14 \begin{tabular}{l|l} 
DOI: 10.1159/000493951 & $\begin{array}{l}\text { O } 2018 \text { The Author(s). Published by S. Karger AG, Basel } \\
\text { www.karger.com/cpb }\end{array}$
\end{tabular} \\ Wang et al.: Different Chemotherapies in Advanced/Metastatic Urothelial Carcinoma}

first-line GC regimen could yield a better survival benefit, but also worsen adverse events for advanced or metastatic UC. This was inclusive and consistent with previous studies. Depending on a comprehensive consideration of survival benefits and side effects, GC or MVAC remained the mainstay of chemotherapy regimens. Clinically, clinicians should take the general medical conditions and the extent of the disease into consideration. Then, they could weigh the merits of these approaches for maximum survival benefit of eligible patients.

\section{Conclusion}

Despite more adverse events, adding a paclitaxel or sorafenib into the first-line GC regimen seemed to have a better effectiveness in the treatment of advanced/metastatic UC and these provided a train of thought for developing the next generation of chemotherapy drugs. More attention should be paid not only to the survival benefits, but also to the increased incidence of adverse events. When considering the survival benefit and the adverse events, GC or MVAC remained the mainstay of chemotherapy regimens. General medical conditions, as well as the extent of the disease, should be taken into consideration by clinicians who could, then, weigh the merits of these approaches to best suit the survival benefits of qualified patients. Larger sample sizes of strictly designed randomized controlled trials were required to validate our findings. Furthermore, the application of this network meta-analysis was anticipated to inform current therapeutic decision-making as well as guide treatment design of the future research.

\section{Acknowledgements}

This article was funded by the Medical key talent of Jiangsu Province [grant number: ZDRCA2016009] and the National Natural Science Foundation of China [grant number: 81501245].

\section{Disclosure Statement}

The authors declare to have no competing interests.

\section{References}

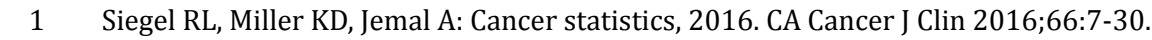

$\rightarrow 2$ Rouanne M, Loriot Y, Lebret T, Soria JC: Novel therapeutic targets in advanced urothelial carcinoma. Crit Rev Oncol Hematol 2016;98:106-115.

3 Von dMH, Sengelov L, Roberts JT, Ricci S, Dogliotti L, Oliver T, Moore MJ, Zimmermann A, Arning M: Longterm survival results of a randomized trial comparing gemcitabine plus cisplatin, with methotrexate, vinblastine, doxorubicin, plus cisplatin in patients with bladder cancer. J Urology 2005;23:4602.

4 Niedersuss-Beke D, Puntus T, Kunit T, Grunberger B, Lamche M, Loidl W, Bohm R, Kraischits N, Kudlacek S, Schramek P, Meran JG: Neoadjuvant Chemotherapy with Gemcitabine plus Cisplatin in Patients with Locally Advanced Bladder Cancer. Oncology 2017;93:36-42.

5 Shelley M, Cleves A, Wilt TJ, Mason M: Gemcitabine for unresectable, locally advanced or metastatic bladder cancer. Cochrane Database Syst Rev 2011;4:CD008976.

-6 Cumberbatch K, He T, Thorogood Z, Gartrell BA: Emerging Drugs for Urothelial (Bladder) Cancer. Expert Opin Emerg Drugs 2017;22:149-164.

7 Yvonne K, Katrin S, Martin B, Julie S, Jan SA, Laura-Maria K: Variant histology in bladder cancer: how it should change the management in non-muscle invasive and muscle invasive disease? Transl Androl Urol 2016;5:692-701. 


\section{Cellular Physiology Cell Physiol Biochem 2018;50:1-14 \begin{tabular}{l|l} 
and Biochemistry Published 10.1159/000493951 2018 & $\begin{array}{l}\text { @) } 2018 \text { The Author(s). Published by S. Karger AG, Basel } \\
\text { www.karger.com/cpb }\end{array}$ \\
\hline
\end{tabular}

Wang et al.: Different Chemotherapies in Advanced/Metastatic Urothelial Carcinoma

8 Aragon-Ching JB, Trump DL: Systemic therapy in muscle-invasive and metastatic bladder cancer: current trends and future promises. Future Oncology 2016;12:2049.

-9 Caldwell DM, Ades AE, Higgins JP: Simultaneous comparison of multiple treatments: combining direct and indirect evidence. BMJ 2005;331:897-900.

10 Lu G, Ades AE: Combination of direct and indirect evidence in mixed treatment comparisons. Stat Med 2004;23:3105-3124.

-11 Salanti G, Higgins JP, Ades AE, Ioannidis JP: Evaluation of networks of randomized trials. Stat Methods Med Res 2008;17:279-301.

12 Higgins J, Green SE: Cochrane Handbook for Systematic Reviews of Interventions Version 5.1.0. Naunyn Schmiedebergs Arch Exp Pathol Pharmakol 2011;2011:S38.

13 Higgins JP, Thompson SG, Deeks JJ, Altman DG: Measuring inconsistency in meta-analyses. Br Med J 2003;327:557-560.

14 Dersimonian R: Meta-analysis in clinical trials. Control Clin Trials 1986;7:177.

15 Egger M, Smith GD, Schneider M, Minder C: Bias in meta-analysis detected by a simple, graphical test. BMJ Brit Med J 1997;315:629-634.

16 gemtc: Network Meta-Analysis Using Bayesian Methods. URL: https://rdrr.io/cran/gemtc/.

17 Lumley T: Network meta-analysis for indirect treatment comparisons. Stat Med 2010;21:2313-2324.

18 Wu HY, Huang JW, Lin HJ, Liao WC, Peng YS, Hung KY, Wu KD, Tu YK, Chien KL: Comparative effectiveness of renin-angiotensin system blockers and other antihypertensive drugs in patients with diabetes: systematic review and bayesian network meta-analysis. BMJ 2013;347:f6008.

19 Zhang X, Shen P, He Q Yin X, Chen Z, Gui H, Shu K, Tang Q, Yang Y, Pan X: Different lasers in the treatment of benign prostatic hyperplasia: a network meta-analysis. Sci Rep 2016;6:23503.

-20 Du S, Ye J, Chen H, Li X, Lin Q: Interventions for Treating 3- or 4-part proximal humeral fractures in elderly patient: A network meta-analysis of randomized controlled trials. Int J Surg 2017;48:240-246.

-21 Wa D, Zhu P, Long Z: Comparative Efficacy and Safety of Anti-Platelet Agents in Cerebral Ischemic Disease: A Network Meta-Analysis. J Cell Biochem 2017; DOI:10.1002/jcb.26065.

22 Qin Z, Wu J, Tian J, Zhou J, Liu Y, Liu Z: Network Meta-Analysis of the Efficacy of Acupuncture, Alphablockers and Antibiotics on Chronic Prostatitis/Chronic Pelvic Pain Syndrome. Sci Rep 2016;6:35737.

23 Gelman A, Rubin DB: Inference from Iterative Simulation Using Multiple Sequences. Stat Sci 1992;7:457472.

24 Dias S, Welton NJ, Caldwell DM, Ades AE: Checking consistency in mixed treatment comparison metaanalysis. Stat Med 2010;29:932-944.

25 Hsieh MC, Huang CH, Chiang PH, Chen YY, Tang Y, Su YL: Tailored Selection of First-Line Cisplatin-Based Chemotherapy in Patients with Metastatic Urothelial Carcinoma of Bladder. J Cancer 2016;7:1347-1352.

-26 Oudard S, Culine S, Vano Y, Goldwasser F, Théodore C, Nguyen T, Voog E, Banu E, Vieillefond A, Priou F: Multicentre randomised phase II trial of gemcitabine + platinum, with or without trastuzumab, in advanced or metastatic urothelial carcinoma overexpressing Her2. Eur J Cancer 2015;51:45-54.

-27 Hussain M, Daignault S, Agarwal N, Grivas PD, Siefkerradtke AO, Puzanov I, Macvicar GR, Levine EG, Srinivas S, Twardowski P: A Randomized Phase 2 Trial of Gemcitabine/Cisplatin With or Without Cetuximab in Patients With Advanced Urothelial Carcinoma. Cancer 2014;120:2684.

-28 Krege S, Rexer H, Vom DF, De GP, Klotz T, Retz M, Heidenreich A, Kühn M, Kamradt J, Feyerabend S: Prospective randomized double-blind multicentre phase II study comparing gemcitabine and cisplatin plus sorafenib chemotherapy with gemcitabine and cisplatin plus placebo in locally advanced and/or metastasized urothelial cancer: SUSE (AUO-AB 31/05). BJU Int 2014;113:429-436.

29 Sternberg CN, Skoneczna IA, Castellano D, Theodore C, Blais N, Voog E, Bellmunt J, Peters F, Leguennec S, Cerbone L: Larotaxel with Cisplatin in the First-Line Treatment of Locally Advanced/Metastatic Urothelial Tract or Bladder Cancer: A Randomized, Active-Controlled, Phase III Trial (CILAB). Oncology 2013;85:208215.

-30 Bellmunt J, Von dMH, Mead GM, Skoneczna I, De SM, Daugaard G, Boehle A, Chevreau C, Pazares L, Laufman LR: Randomized phase III study comparing paclitaxel/cisplatin/gemcitabine and gemcitabine/cisplatin in patients with locally advanced or metastatic urothelial cancer without prior systemic therapy: EORTC Intergroup Study 30987. J Clin Oncol 2012;30:1107. 


\section{Cellular Physiology Cell Physiol Biochem 2018;50:1-14 \begin{tabular}{l|l} 
and Biochemistry Published 10.1159/000493951 2018 & $\begin{array}{l}\text { ¿ } 2018 \text { The Author(s). Published by S. Karger AG, Basel } \\
\text { www.karger.com/cpb }\end{array}$
\end{tabular} \\ Wang et al.: Different Chemotherapies in Advanced/Metastatic Urothelial Carcinoma}

-31 Bamias A, Dafni U, Karadimou A, Timotheadou E, Aravantinos G, Psyrri A, Xanthakis I, Tsiatas M, Koutoulidis V, Constantinidis C: Prospective, open-label, randomized, phase III study of two dose-dense regimens MVAC versus gemcitabine/cisplatin in patients with inoperable, metastatic or relapsed urothelial cancer: a Hellenic Cooperative Oncology Group study (HE 16/03). Ann Oncol 2013;24:1011-1017.

-32 Dogliotti L, Carteni G, Siena S, Bertetto O, Martoni A, Bono A, Amadori D, Onat H, Marini L: Gemcitabine plus cisplatin versus gemcitabine plus carboplatin as first-line chemotherapy in advanced transitional cell carcinoma of the urothelium: results of a randomized phase 2 trial. Eur Urol 2007;52:134-141.

-33 Roberts JT, von der Maase H, Sengelov L, Conte PF, Dogliotti L, Oliver T, Moore MJ, Zimmermann A, Arning M: Long-term survival results of a randomized trial comparing gemcitabine/cisplatin and methotrexate/ vinblastine/doxorubicin/cisplatin in patients with locally advanced and metastatic bladder cancer. Ann Oncol 2006;17:v118-122.

-34 Bamias A, Aravantinos G, Deliveliotis C, Bafaloukos D, Kalofonos C, Xiros N, Zervas A, Mitropoulos D, Samantas E, Pectasides D, Papakostas P, Gika D, Kourousis C, Koutras A, Papadimitriou C, Bamias C, Kosmidis P, Dimopoulos MA, Hellenic Cooperative Oncology G: Docetaxel and cisplatin with granulocyte colony-stimulating factor (G-CSF) versus MVAC with G-CSF in advanced urothelial carcinoma: a multicenter, randomized, phase III study from the Hellenic Cooperative Oncology Group. J Clin Oncol 2004;22:220-228.

35 Dreicer R, Manola J, Roth BJ, See WA, Kuross S, Edelman MJ, Hudes GR, Wilding G: Phase III trial of methotrexate, vinblastine, doxorubicin, and cisplatin versus carboplatin and paclitaxel in patients with advanced carcinoma of the urothelium. Cancer 2004;100:1639-1645.

-36 Lorusso V, Crucitta E, Silvestris N, Rosati G, Manzione L, De Lena M, Palmeri S, Gebbia V, Mancarella S, Sobrero A, Pezzella G, Comella P, Mangiameli A, Muci D: Randomised, open-label, phase II trial of paclitaxel, gemcitabine and cisplatin versus gemcitabine and cisplatin as first-line chemotherapy in advanced transitional cell carcinoma of the urothelium. Oncol Rep 2005;13:283-287.

-37 Lehmann J, Retz M, Steiner G, Albers P, Jaeger E, Knuth A, Lippert C, Koser M, Stockamp K, Otto C, Melchior H, Fassmann C, Potratz C, Loch T, Derigs HG, Becker T, Kalble T, Piechota HJ, Hertle L, Weinknecht S, et al.: [Gemcitabine/cisplatin vs. MVAC. 5 year survival outcome of the phase III study of chemotherapy of advanced urothelial carcinoma in Germany]. Urologe A 2003;42:1074-1086.

-38 von der Maase H, Hansen SW, Roberts JT, Dogliotti L, Oliver T, Moore MJ, Bodrogi I, Albers P, Knuth A, Lippert CM, Kerbrat P, Sanchez Rovira P, Wersall P, Cleall SP, Roychowdhury DF, Tomlin I, Visseren-Grul CM, Conte PF: Gemcitabine and cisplatin versus methotrexate, vinblastine, doxorubicin, and cisplatin in advanced or metastatic bladder cancer: results of a large, randomized, multinational, multicenter, phase III study. J Clin Oncol 2000;18:3068-3077.

-39 Scott B. Saxman KJP, Lawrence H. Einhorn, E. David Crawford, lan Tannock, Derek Raghavan, Patrick J. Loehrer, Sr, and Donald Trump: Long-Term Follow-Up of a Phase III Intergroup Study of Cisplatin Alone or in Combination With Methotrexate, Vinblastine, and Doxorubicin in Patients With Metastatic Urothelial Carcinoma: A Cooperative Group Study. J Clin Oncol 1997;15:2564-2569.

-40 Patrick J. Loehrer S, Lawrence H. Einhorn, Paul J. Elson, E. David Crawford, Philip Kuebler, Ian Tannock, Derek Raghavan, Robin Stuart-Harris, Michael F. Sarosdy: A Randomized Comparison of Cisplatin Alone or in Combination With Methotrexate, Vinblastine, and Doxorubicin in Patients With Metastatic Urothelial Carcinoma: A Cooperative Group Study. J Clin Oncol 1992;10:1066-1073.

-41 Sternberg CN, Yagoda A, Scher HI, Watson RC, Geller N, Herr HW, Morse MJ, Sogani PC, Vaughan ED, Bander $\mathrm{N}$ : Methotrexate, vinblastine, doxorubicin, and cisplatin for advanced transitional cell carcinoma of the urothelium. Efficacy and patterns of response and relapse. Cancer 1989;64:2448.

42 Giannatempo P, Pond GR, Sonpavde G, Raggi D, Naik G, Galsky MD, Bellmunt J, Necchi A: The Impact of Adding Taxanes to Gemcitabine and Platinum Chemotherapy for the First-Line Therapy of Advanced or Metastatic Urothelial Cancer: A Systematic Review and Meta-analysis. Eur Urol 2016;69:624-633.

43 Necchi A, Pond GR, Raggi D, Giannatempo P, Vogelzang NJ, Grivas P, Galsky MD, Bellmunt J, Sonpavde G: Efficacy and Safety of Gemcitabine Plus Either Taxane or Carboplatin in the First-Line Setting of Metastatic Urothelial Carcinoma: A Systematic Review and Meta-Analysis. Clin Genitourin Cancer 2017;15:23-30.e2.

-44 Nakaoka H, Inoue I: Meta-analysis of genetic association studies: methodologies, between-study heterogeneity and winner's curse. J Hum Genet 2009;54:615-623. 